\title{
Geophysikalische Einzelschriften
}

\author{
Herausgeber: Prof. Dr. Paul Raethjen
}

Direktor des Geophysikalischen Instituts der Universität Hamburg

Heft 3

\author{
Kurzer Abriß der \\ Meteorologie
}

dynamisch gesehen

TEIL III

Zirkulationen der Atmosphäre

von

Paul Raethjen

Hamburg 1954

Selbstverlag des Heraugqebers

Die Geophysikaliseben Einzelschriften kơnen vom Goophysikalischen Institut. Hamburg 13. Rothenbaumchausaee 80 bezoget worden.

Bisher ergehienen 4
Notdruds: Kongtitution der Atmosphăre (Kurzer Abriß Teil I, 1947) ron P. Baethjen (100 Seiten) DM 3.50

Notdruds: Konstitution der Atmosphăre (Kurzer Abriß Teil I, 1947) von P. Raethjen (100 Seiten) DM 3.50
Heft 1: $\begin{array}{ll}\text { Heft 1: } & \text { Wrrmehaushalt der Atmogphate (Kurzer Abriß Teil II, 1950) von P. Raethjen (52 Seiten) DM 3.00 } \\ \text { Heft 2: Frontslzytlogenesis (1954) ron P. Raethjon (16 Seiten) } & \text { DM 1.00 }\end{array}$ $\begin{array}{lll}\text { Heft 2: Frontalzylogenesis (1954) ron P. Raethjon (16 Seiten) } & \text { DM } 1.00 \\ \text { Heft 3: } & \text { Zirkulationen der Atmogphäro (Kurzer Abriß Teil III, 1954) von P. Haethjen (62 Seiten) DM 4.00 }\end{array}$ Postschedzonto: Hamburg, Konto-Nr. 9985 "Geophysikalische Einzelsehriften. Hauburg 13" 


\section{Gedrudkt mit Linterstũtzung der Joachim-Jungius-Gesellschaft der Wissenschaften e. V.. Hamburg}

Copvright 1954 by Prof. Dr. Paul Raethien. Hamburer 13. Rothenbaumchaussee 80) 